\title{
The ethics of new technologies to track drug adherence
}

— Cite as: CMAJ 2018 October 9;190:E1209-10. doi: 10.1503/cmaj.171514

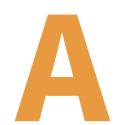
bilify MyCite, a pill that includes a digital sensor that can track medication adherence, was approved by the US Food and Drug Administration in November 2017. ${ }^{1}$ The pill's sensor communicates with an external patch, which transmits information on whether the pill has been ingested to a patient's mobile devices so that they can track their medication intake. It also allows health care workers to access this information to assist the patient in adhering to medication regimens. It has been approved for use in patients with schizophrenia and bipolar disorder. Its use for other diseases that require careful medication adherence is already being considered. . $^{2,3}$

The capacity of Abilify MyCite and similar technologies ${ }^{2}$ to share information with third parties creates an opportunity to improve medication adherence and sometimes the public's health. However, these technologies raise serious privacy concerns around who will and should have access to this information. Because these technologies are growing in popularity but have not yet been approved by Health Canada, we should take the opportunity to consider their potential use and implementation within Canada.

The arguments in favour of such technologies centre on the potential lack of adherence to drug regimens that not only put the individual patient at risk but may also threaten the public's health or security. ${ }^{2,3}$ For example, treatment of tuberculosis (TB) relies on directly observed therapy to promote adherence to drug regimens. Directly observing the patient taking the required medication allows caregivers to monitor for drug adverse effects and, purportedly, reduce transmission of disease. Technologies that facilitate such tracking can be described as a mere evolution of these previously accepted techniques to monitor adherence.



The argument against these devices centres on concerns about privacy and confidentiality, namely, that these devices collect data about adherence that are linked to specific individuals. ${ }^{2,3}$ This harvesting of data, combined with its cloudbased storage, creates concerns about security breaches. Another issue is that patients do not have a real choice to say "no" to this technology, either because a health care worker can directly mandate its use (e.g., under mental or public health laws) or because indirect pressure may make a patient choose to use such devices despite having reservations (e.g., threat of involuntary isolation). At the core of such arguments lie concerns about the erosion of autonomy and, with it, a trend toward more paternalistic health policies.

A key ethical tension in the use of such adherence devices can be described as a tension between what is good for the public's health and the patient's autonomy, control over their health information, and right to choose what does and does not occur to their bodies. However, we argue that a more nuanced understanding of autonomy can lead to better public health policy that still protects the individual patient. Moving beyond the often false and tired dichotomy of the public's health versus the patient's choices ${ }^{4}$ allows us to think of new ways of regulating and using drug adherence devices. 


\section{Autonomy is misunderstood}

Autonomy is often invoked as meaning that each person should be allowed to do as they please (so long as they do not hurt others) and, in the context of clinical medicine, that each person should be allowed to decide what happens to their bodies during care. Although such simplicity in conceptualization and application has many advantages, autonomy is much more complex, both theoretically and practically. Autonomy usually means that a person is self-directed; i.e., they can direct their life toward certain ends and away from others. To exercise this autonomy in certain situations, simple noninterference is sufficient. But autonomy is often much more than simply not interfering in the lives of others and with their choices. ${ }^{5}$ To get a fuller sense of autonomy, we ought to consider the importance of making informed choices, how someone becomes autonomous, and how much of what makes people autonomous lies outside their direct control.

First, to be truly autonomous, it matters that a person makes informed decisions about how their lives are shaped. ${ }^{6}$ Sometimes a person might make an informed choice to remove choices and agree to outside interference. For example, a person on a diet with a predilection for sweets might request that their family members not bring home sugary treats to remove any temptations. The key in such situations is that the person retains the freedom to remove the self-imposed limitation.

Second, people do not become autonomous or act autonomously in a vacuum; we learn to be autonomous in relation to others. ${ }^{7}$ For example, people become autonomous adults by learning how to be autonomous from their family and friends, which includes learning about and then challenging their family's values based on other influences (e.g., reading the news). Likewise, most people are aware that their actions have repercussions for others and behave accordingly. Stated differently, my autonomous choices influence others' abilities to act autonomously and, as such, we must all act in such a way that allows others to be autonomous. ${ }^{8}$

Third, how autonomous people are depends greatly on external factors. ${ }^{5}$ From obviously important external pressures (e.g., war, famine or being born into wealth) to less obvious and, perhaps somewhat triv- ial, pressures (e.g., the size of soda cups), not merely our choices but how we direct our lives are constantly being shaped by external factors. Concerns about justice can potentially be redescribed as a means of rectifying those social, political and economic factors that are deemed morally wrong and impinge on autonomy.

The public health arguments in favour of adherence devices centre on their ability to help health care workers protect the health of the patient and the public, such as with other types of surveillance activities. Each person benefits from collective public health efforts, including surveillance. ${ }^{5}$ The individual-level benefits of surveillance justify those external factors that positively affect autonomy. Better surveillance can help prevent the spread of infectious diseases and can help manage resources in cases of chronic diseases, all of which allows individuals to be more selfdirecting by promoting stewardship and social protections.

Even if a person prefers not to be monitored for drug adherence, individual autonomy must be balanced with the autonomy of others. ${ }^{8}$ People might need to accept some form of drug adherence monitoring as beneficial to everyone's ability to be selfdirecting. In other words, if autonomy is held as an important value or principle in health care and public health, then a commitment is made to abide sometimes by public health actions that improve the capacity of people to be autonomous.

Of course, such a robust sense of autonomy would also lead to restrictions on how drug adherence devices should be used in practice. First and foremost, their involuntary use should only be contemplated if there is an actual threat to public health. For example, adherence to medication in the case of psychiatric conditions should be voluntary and free of any pressure from health care workers because the benefit is primarily to the individual client (although this would still leave room for strongly advising clients ${ }^{9}$ and for cases where a client may be deemed temporarily incompetent).

More importantly, we must guard against unjust and discriminatory differential uses of drug adherence devices for the purported benefit of the public's health. We must ensure that we do not contemplate using drug adherence devices only for diseases that predominately affect those of lower socioeconomic status. For example, some have argued that the reason directly observed therapy gained such unquestioned traction in monitoring drug adherence in the case of TB is that TB overwhelmingly affects people of lower socioeconomic status who are unable to safely express any reservations because they are socially and politically marginalized. ${ }^{10}$

Canada has the opportunity to be proactive and carefully examine the allowable uses of drug adherence technologies, both for the sake of the public's health and for its use in clinical settings. In doing so, Canada must guard against overly simplistic arguments that pit the public good versus that of the private person and, instead, think of how to regulate these devices in a way that underscores the important relationship between an individual's autonomy and their place within society.

\section{Diego S. Silva PhD}

Faculty of Health Sciences, Simon Fraser University, Burnaby, BC

\section{Jeremy Snyder PhD}

Faculty of Health Sciences, Simon Fraser

University, Burnaby, BC

\section{References}

1. Highlights of prescribing information: Abilify MyCite. Washington (DC): US Food and Drug Administration; 2017. Available: www.accessdata. fda.gov/drugsatfda_docs/label/2017/207202lbl.pdf (accessed 2017 Dec. 22).

2. Belluck P. First digital pill approved to worries about 'Big Brother'. The New York Times. Available: www.nytimes.com/2017/11/13/health/digital-pill -fda.html (accessed 2017 Dec. 22).

3. Lee BY. FDA approves first digital pill that you (and others) can track. Forbes 2017 Nov. 13. Available: www.forbes.com/sites/brucelee/2017/11/13 /fda-approved-first-digital-pill-that-you-and-others -can-track/\#3694923e6419 (accessed 2017 Dec. 22).

4. Coggon J. What makes health public? A critical evaluation of moral, legal, and political claims in public health. New York: Cambridge University Press; 2012.

5. Wilson J. The right to public health. J Med Ethics 2016;42:367-75.

6. Dan-Cohen M. Conceptions of choice and conceptions of autonomy. Ethics 1992;102:221-43.

7. Wardrope A. Relational autonomy and the ethics of health promotion. Public Health Ethics 2014;8:50-62.

8. Kant I. The metaphysics of morals. In: Gregor MJ, editor. The Cambridge Edition of the Works of Immunel Kant: Practical Philosophy. New York: Cambridge University Press; 1996.

9. Mill JS. On liberty. New York: W.W. Norton \& Company; 1975.

10. Coker RJ. From chaos to coercion: detention and the control of tuberculosis. New York: St. Martin's Press; 2000.

This article has been peer reviewed. 